\title{
Fuzzy Edge Linking Process on Fuzzy Noise Filtered Image
}

\author{
G.Sudhavani \\ Associate Professor, \\ Department of ECE, \\ RVR \& JC College of \\ Engineering, Guntur.
}

\author{
S.Sravani \\ Student (M.Tech), \\ Department of ECE, \\ RVR \& JC College of \\ Engineering, Guntur.
}

\author{
P.Venkateswara Rao \\ Associate Professor, \\ Department of CSE, \\ RVR \& JC College of \\ Engineering, Guntur.
}

\author{
K.Satya Prasad \\ Professor, \\ Department of ECE, \\ JNTU Kakinada, Kakinada.
}

\begin{abstract}
Processing of images plays a vital role in many fields such as medical and scientific applications. During the transmission of images, effect of noise plays a key role. A fuzzy filter is presented for additive noise removal from color images. During the process of noise removal, some of the edges may be disappeared. This paper presents two independent fuzzy based edge linking algorithms which are capable of finding a set of edge points in an image and linking these edge points by thresholding. The first algorithm includes a set of 16 fuzzy templates, representing the edge profiles of different types. The second algorithm relies on the image gradient to locate breaks in uniform regions and is based on fuzzy if-then rules. Performance evaluation of these algorithms is known by calculating peak signal to noise ratio (PSNR).
\end{abstract}

\section{Keywords}

Fuzzy filter, Fuzzy rule - based system, Additive noise, Gradient, Edge detection, Intuitionistic fuzzy set, Membership degree, Hesitation degree, Intuitionistic fuzzy divergence, Fuzzy interference system.

\section{INTRODUCTION}

Image processing is a growing research field. Images have always played an important and essential role in human life, not only as a way to communicate, but also for other applications like commercial, industrial and scientific. In this paper two independent fuzzy edge linking techniques are presented that efficiently link the edge points of noise filtered image.

Especially there are three types of noise exist: Gaussian noise, Salt and pepper noise, Speckle noise. Gaussian noise is most commonly used as Additive White Gaussian Noise (AWGN). Salt and pepper noise itself as randomly occurring white and black pixels. Speckle noise is also known as multiplicative noise, i.e. the gray levels of speckle noisy pixels are in direct proportion to the local gray levels in any area of an image.

Distance computation plays an important role in removal of noise from color images. The distance between two couples is calculated according to the Minkowski's distance. To compute scaling factors of the filter, Takagi-sugeno fuzzy model is used. The concept behind the evolution of fuzzy rules for the additive noise removal is to assign large scaling factors to the neighbors that have similar colors as the centre pixel.

Fuzzy set theory and fuzzy logic [1] offers powerful tools to describe and process human knowledge. Some of the fuzzy filters designed earlier for noise reduction are a new fuzzy filter for image enhancement [2], noise adaptive soft switching median filter [3], noise reduction by fuzzy image filtering [4], fuzzy two step color filter [5], a fuzzy impulse noise detection and reduction method [6], and so on. The different methods using fuzzy filters for reduction of noise are explained in the literature [7], [8]. Most of the current fuzzy noise reduction techniques do not produce convincing results for additive noise and introduces distortions, especially on edge or texture elements. Therefore this paper presents two independent fuzzy techniques for detecting and linking the edge points of noise filtered images.

Edge linking is a critical part of many computer vision systems. The edge serves in simplifying the analysis of images by drastically reducing the data to be processed and preserving the useful structural information about object boundaries. An edge detector produces either a set of edge points or edge fragments. Edge detection provides a means of segmenting [9] the image in to meaningful regions. There are many fuzzy models [10] that attempt to locate regions in images that are related to edges. Edge linking techniques attempt to bind the detected edge fragments, forming an image with better visual clarity.

In this paper, a new attempt has been made to use the Intuitionistic fuzzy set theory in the image edge linking. Intuitionistic fuzzy set takes into account the uncertainty in assignment of membership degree known as hesitation degree. A new definition of distance measure, called Intuitionistic fuzzy divergence (IFD) is used. Unlike fuzzy divergence that considers only the membership degree [11], [12], IFD considers not only the membership degree, but also the nonmembership degree, and the hesitation degree.

A digital color image $X$ can be represented by a 2-D array of vectors where an address $(k, l)$ defines a position in $X$, called a pixel or picture element. If $X(k, l, 1)$ denotes the red component, $X(k, l, 2)$ and $X(k, l, 3)$ denote the green and 
blue components of a pixel at position $(k, l)$ respectively in an (noise - free) image $X$, then the noisy color image $N I$ at position $(k, l)$ can be denoted as follows:

$$
\begin{aligned}
& {[N I(k, l, 1) N I(k, l, 2) N I(k, l, 3)]=} \\
& {\left[\left(X(k, l, 1)+\partial_{1}\right)\left(X(k, l, 2)+\partial_{2}\right)\left(X(k, l, 3)+\partial_{3}\right)\right]}
\end{aligned}
$$

With $\partial_{1}, \partial_{2}$ and $\partial_{3}$ are three separate randomly Gaussian distributed values with means $\left(\mu_{1}, \mu_{2}\right.$ and $\left.\mu_{3}\right)$ and standard deviations $\left(\sigma_{1}, \sigma_{2}\right.$ and $\left.\sigma_{3}\right)$ respectively. $N I$ is the color image that is corrupted with additive white Gaussian noise.

\section{FUZZY NOISE FILTER}

While capturing or transmission of images the effect of noise may degrade the quality of the image so it is desirable to perform some sort of noise reduction on an image. The conventional idea for noise reduction is to average a pixel using other pixel values from its neighborhood. The fuzzy noise filter consists of two sub filters. The first sub filter calculates fuzzy distances among the color components of the central pixel and its neighborhood. These distances determine in what extent each component should be corrected. The aim of the second sub filter is to calculate the color components differences to retain the fine details of the image. The difference between this proposed filter and other vector based approaches as [13],[14] is that instead of calculating the 3-D distances between pixels, the three 2-D distances are used together with three fuzzy rules to calculate the weights used for the Takagi-Sugeno fuzzy model [15].

\subsection{First Sub Filter with Fuzzy Rules}

The red, green, and blue component at a certain pixel position of a noisy image $N I$ is denoted as $N I(k, l, 1), N I(k, l, 2)$ and $N I(k, l, 3)$, respectively. So, for each pixel position, there are three components that describe the color. For each pixel position $(k, l)$ the following pairs are determined: the pair red and green denoted as $R G(k, l)=(N I(\mathrm{k}, 1,1), N I(k, l, 2))$ the pair red and blue denoted as $R B(k, l)=(N I(\mathrm{k}, 1,1), N I(k, l, 3))$ and the pair green and blue denoted as $G B(k, l)=(N I(\mathrm{k}, 1,2), N I(k, l, 3))$. To filter the current image pixel, a window of size $(2 I+1) \times(2 I+1)$ centered at position $(k, l)$ is used. Next certain scaling factors are assigned to each of the pixels in window. The scaling factors $w(k+i, l+j, 1), \quad w(k+i, l+j, 2) \quad$ and $w(k+i, l+j, 3) \quad$ are for the red, green and blue components at position $(k+i, l+j)$ respectively. These scaling factors are assigned according to the following fuzzy rules.
Fuzzy rule 1: The first fuzzy rule defines the weight $w(k+i, l+j, 1)$ for the red component of the neighbor $N I(k+i, l+j, 1)$, i.e.

IF the distances are small between the pairs $R G(k, l)$, $R G(k+i, l+j)$ and $R B(k, l), R B(k+i, l+j)$ THEN the scaling factor $w(k+i, l+j, 1)$ is large.

Similarly, the Fuzzy rule 2 and Fuzzy rule 3 [16] defines the weight for green and blue component respectively.

The concept behind these simple fuzzy rules is to assign large scaling factors to the neighbors that have similar colors as the center pixel. The distance between two couples is calculated according to the Minkowski's distances. This is illustrated in equation (2) for red-green pair

$$
\begin{aligned}
& D(R G(k, l), R G(k+i, l+j))= \\
& {\left[\begin{array}{c}
(N I(k+i, l+j, 1)-N I(k, l, 1))^{2}+ \\
(N I(k+i, l+j, 2)-N I(k, l, 2))^{2}
\end{array}\right]^{1 / 2}}
\end{aligned}
$$

To compute the value that expresses the degree to which the distances of two couples is small, a fuzzy set small is used. From the membership functions of the fuzzy set small the corresponding membership degrees can be derived. If the distance between two couples has a membership degree one (zero) in fuzzy set small, it means that this distance is considered as (not) small for sure. Membership degrees between zero and one indicate that there is some kind of uncertainty in determining whether the distance is small or not.

Three such fuzzy sets are defined one for each pair. All these fuzzy sets depend on the parameters denoted as $P_{R G}$, $P_{R B}$ and $P_{G B}$. The parameter $P_{R G}$ is determined as follows:

$$
P_{R G}(k, l)=\max _{k, l \in \Omega}\left(\gamma_{R G}(k, l, i, j)\right)
$$

Where $\Omega$ defines the $(2 I+1) \times(2 I+1)$ neighborhood around the central pixel, i.e. $i, j \in\{-I,-I+1, \ldots, 0, \ldots, I-1, I\}, \quad$ and where $\gamma_{R G}(k, l, i, j), \gamma_{R B}(k, l, i, j)$ and $\gamma_{G B}(k, l, i, j)$ were used as convenient notation for the distances. (i.e., $\gamma_{R G}(k, l, i, j)=D(R G(k, l), R G(k+i, l+j)), \quad$ similarly for $\gamma_{R B}(k, l, i, j)$ and $\left.\gamma_{G B}(k, l, i, j)\right)$.

So, the parameters (for the spatial position $(k, l)) P_{R G}(k, l), P_{R B}(k, l)$ and $P_{G B}(k, l)$ are equal to the maximal distance between the red-green, 
red-blue and green-blue pairs in a $(2 I+1) \times(2 I+1)$ neighborhood around the centre $\operatorname{pixel}(k, l)$.

The $\gamma$ 's of expression (4) are used to calculate the scaling factors as introduced by fuzzy rules. In these rules, the intersection of two fuzzy sets is specified by mapping $T$-norms. This means for instance that the fuzzification of the antecedent of fuzzy rule 1 is $\mu_{\text {small } 1}\left(\gamma_{R G}(k, l, i, j)\right) \square \mu_{\text {small } 2}\left(\gamma_{R B}(k, l, i, j)\right)$, where $\mu_{\text {small } 1}$ and $\mu_{\text {small } 2}$ are equal to membership function small, shown in expression (2), with parameters $P_{R G}(k, l)$ and $P_{R B}(k, l), \quad$ respectively. The obtained value $\left(\mu_{\text {small } 1}\left(\gamma_{R G}(k, l, i, j)\right) \square \mu_{\text {small } 2}\left(\gamma_{R B}(k, l, i, j)\right)\right) \quad$ is called the activation degree of the fuzzy rule and is used to obtain corresponding scaling factor, i.e. $w(k+i, l+j, 1)=\mu_{\text {small } 1}\left(\gamma_{R G}(k, l, i, j)\right) \square \mu_{\text {small } 2}\left(\gamma_{R B}(k, l, i, j)\right)$ similarly the remaining two scaling factors are calculated by using activation degrees.

Where $\mu_{\text {small } 1}, \mu_{\text {small } 2}$ and $\mu_{\text {small } 3}$ are equal to membership function small ,shown in expression (2), with parameters $P_{R G}(k, l), \quad P_{R B}(k, l) \quad$ and $\quad P_{G B}(k, l)$ respectively. The output of the first sub filter can finally be illustrated for the red component, where the output image is denoted as $A$, i.e.,

$$
A(k, l, 1)=\frac{\sum_{i=-I}^{+I} \sum_{j=-I}^{+I} w(k+i, l+j, 1) \square N I(k+i, l+j, 1)}{\sum_{i=-I}^{+I} \sum_{j=-I}^{+I} w(k+i, l+j, 1)}
$$

The filtering process for the green and blue component is similar to the one above.

\subsection{Second Sub Filter to Retain Fine \\ Details}

The second sub filter is a complementary filter to the first one. The purpose of this filter is to improve the first method by reducing the noise in the color components differences without deteriorating the fine details of the image. The second part of the fuzzy filter is realized by taking the local differences in the red, green, and blue environment separately. These local differences are used to calculate the local estimation of the center pixel by taking the average of those differences. Similar to the first part of the filter a window of size $(2 J+1) \times(2 J+1)$ is used where $J$ is not necessarily equal to $I$, centered at $(k, l)$ to filter the current image pixel at position $(k, l)$. Next, the differences (also called gradients or derivatives) are calculated for each element of the window denoted as $B D_{R}, B D_{G}$ and $B D_{B}$ for the red, green, and blue environment respectively. If the output image of the first sub filter is denoted as $\mathrm{A}$, then the local difference for red component is calculated by using (5). Similarly the remaining differences are calculated.

$$
B D_{R}(i, j)=\mathrm{A}(k+i, l+j, 1)-A(k, l, 1)
$$

For all $i, j \in\{-J, \ldots, 0, \ldots,+J\}$. These differences are finally combined to calculate the following correction terms:

$$
e(i, j)=\frac{1}{3}\left(B D_{R}(i, j)+B D_{G}(i, j)+B D_{B}(i, j)\right)
$$

i.e., the average of the difference for the red, green, and blue component is calculated at the same position.

Finally, the output of the second filter, denoted as B, for red component is determined as follows. Like red component the outputs for green and blue components are determined.

$$
B(k, l, 1)=\frac{\sum_{i=-J}^{+J} \sum_{j=-J}^{+J}(A(k+i, l+j, 1)+e(i, j))}{(2 J+1)^{2}}
$$

With $B(k, l, 1)$ is the red component of the output image and where $e(i, j)$ is the correction term for the neighboring pixel $A(k+i, l+j, 1)$.

\section{FUZZY EDGE LINKING}

The objective of denoising is to remove the noise effectively while preserving the original image details as much as possible. Even though the above fuzzy filter retains fine details, some of the edges are not recovered. Hence for further fine recovery of edges, two different fuzzy edge linking algorithms are presented. Many edges are (visually) fragments of larger edge structures. Fuzzy edge linking techniques attempt to bind edge fragments, forming an image with better visual acuity. These two algorithms are applied in a parallel way on the output image of the fuzzy noise filter.

\subsection{First Algorithm}

An edge is a property of an individual pixel and is calculated from the functional behavior of an image in the neighborhood of a pixel. In this algorithm, using intuitionistic fuzzy set [17], a new distance measure called intuitionistic fuzzy divergence (IFD) is proposed, where the three parameters, namely, the membership degree, the non-membership degree, and the hesitation degree are considered. Considering the hesitation degree, the range of membership degree of the two intuitionistic fuzzy sets A and B may be represented as $\left\{\left(\mu_{A}(X),{ }_{A}(X)+\pi_{A}(X)\right)\right\}$,

$\left\{\left(\mu_{B}(X),{ }_{B}(X)+\pi_{B}(X)\right)\right\}$ where $\mu_{A}(X)$ and $\mu_{B}(X)$ are the membership values, $v_{A}(X)$ and $v_{B}(X)$ are the non membership values and $\pi_{A}(X), \pi_{B}(X)$ are the hesitation degrees in the respective sets, with $\pi_{A}(X)=1-\mu_{A}(X)-v_{A}(X) \quad$ and

$\pi_{B}(X)=1-\mu_{B}(X)-v_{B}(X)$.The interval is due to the hesitation in assigning membership values. The distance measure proposed in this algorithm takes in to account the hesitation degrees. 


\subsubsection{Intuitionistic fuzzy divergence}

For two images A and B at $(i, j)^{\text {th }}$ pixels i.e. $a_{i j}$ and $b_{i j}$ of the image, the information discrimination is given as follows:

1. Due to $\mu_{A}\left(a_{i j}\right)$ and $\mu_{B}\left(a_{i j}\right)$ of the $(i, j)^{t h}$ of the image:

$$
e^{\mu_{A}\left(a_{i j}\right)} / e^{\mu_{\mathrm{B}}\left(b_{i j}\right)}=e^{\mu_{A}\left(a_{i j}\right)-\mu_{\mathrm{B}}\left(b_{i j}\right)}
$$

2. Due to $\mu_{A}\left(a_{i j}\right)+\pi_{A}\left(a_{i j}\right)$ and $\mu_{B}\left(a_{i j}\right)+$ of $\pi_{B}\left(b_{i j}\right)$ of the $(i, j)^{t h}$ of the image:

$$
e^{\mu_{A}\left(a_{i j}\right)+\pi_{A}\left(a_{i j}\right)} / e^{\mu_{B}\left(b_{i j}\right)+\pi_{B}\left(b_{i j}\right)}
$$

Corresponding to fuzzy entropy the divergence (Div) between images A and B may be expressed as

$$
D_{1}(A, B)=\sum_{i} \sum_{j}\left[\begin{array}{l}
1-\left(1-\mu_{A}\left(a_{i j}\right)\right) e^{\left(\mu_{A}\left(a_{i j}\right)-\mu_{B}\left(b_{i j}\right)\right)} \\
-\mu_{A}\left(a_{i j}\right) e^{\left(\mu_{B}\left(b_{i j}\right)-\mu_{A}\left(a_{i j}\right)\right)}
\end{array}\right]
$$

Similarly the divergence of B against A may be given as:

$$
D_{1}(B, A)=\sum_{i} \sum_{j}\left[\begin{array}{l}
1-\left(1-\mu_{B}\left(b_{i j}\right)\right) e^{\left(\mu_{B}\left(b_{i j}\right)-\mu_{A}\left(a_{i j}\right)\right)} \\
-\mu_{B}\left(b_{i j}\right) e^{\left(\mu_{A}\left(a_{i j}\right)-\mu_{B}\left(b_{i j}\right)\right)}
\end{array}\right]
$$

So, the total divergence between the pixels $a_{i j}$ and $b_{i j}$ of the images $\mathrm{A}$ and $\mathrm{B}$ due to $\mu_{A}\left(a_{i j}\right)$ and $\mu_{B}\left(a_{i j}\right)$ may be written as

Div $-1(\mathrm{~A}, \mathrm{~B})=\mathrm{D}_{1}(\mathrm{~A}, \mathrm{~B})+\mathrm{D}_{1}(\mathrm{~B}, \mathrm{~A})=$

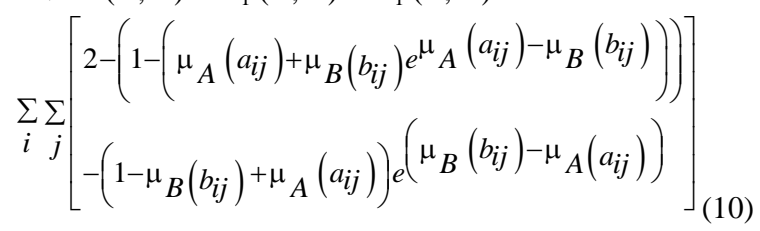

In a likewise manner, the divergence between $a_{i j}$ and $b_{i j}$ of the images $\mathrm{A}$ and $\mathrm{B}$ due to membership values $\mu_{A}\left(a_{i j}\right)+\pi_{A}\left(a_{i j}\right)$ and $\mu_{B}\left(a_{i j}\right)+$ of $\pi_{B}\left(b_{i j}\right)$ may be given as:

$\operatorname{Div}-2(\mathrm{~A}, \mathrm{~B})=$

$$
\sum_{i} \sum_{j}\left[\begin{array}{l}
2-\left(\left[\begin{array}{l}
{\left[-\left(\mu_{A}\left(a_{i j}\right)-\mu_{B}\left(b_{i j}\right)+\pi_{B}\left(b_{i j}\right)-\pi_{A}\left(a_{i j}\right)\right)\right.} \\
\left(\mu_{A}\left(a_{i j}\right)-\mu_{B}\left(b_{i j}\right)\right)-\pi_{B}\left(b_{i j}\right)-\pi_{A}\left(a_{i j}\right)
\end{array}\right)\right. \\
-\left(\begin{array}{l}
\left.1-\left(\pi_{B}\left(b_{i j}\right)-\pi_{A}\left(a_{i j}\right)+\mu_{A}\left(a_{i j}\right)-\right) \mu_{B}\left(b_{i j}\right)\right] \\
\pi_{B}\left(b_{i j}\right)-\pi_{A}\left(a_{i j}\right)-\mu_{A}\left(a_{i j}\right)-\mu_{B}\left(b_{i j}\right)
\end{array}\right)
\end{array}\right]_{(11)}
$$

Thus the Intuitionistic Fuzzy Divergence (IFD), between the images $\mathrm{A}$ and $\mathrm{B}$ due to the membership interval range using (10) and (11) may be written as:
$\mathrm{D}_{\mathrm{H}}(\mathrm{A}, \mathrm{B})$

$$
\begin{aligned}
& \int 2-\left[1-\mu_{A}\left(a_{i j}\right)+\mu_{B}\left(b_{i j}\right)\right] e^{\mu_{A}}\left(a_{i j}\right)-\mu_{B}\left(b_{i j}\right) \\
& -\left[1-\mu_{B}\left(b_{i j}\right)+\mu_{A}\left(a_{i j}\right)\right] e^{\left(\mu_{B}\left(b_{i j}\right)-\mu_{A}\left(a_{i j}\right)\right),}
\end{aligned}
$$

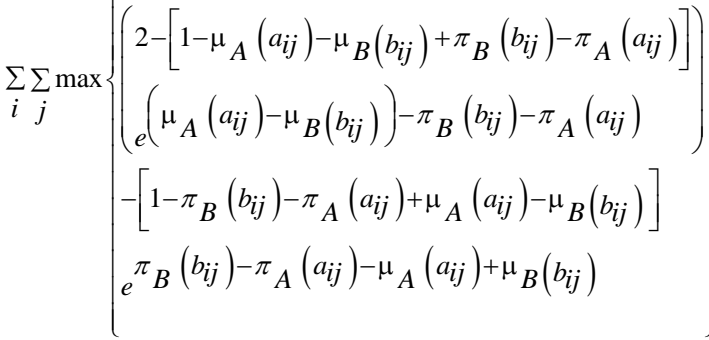

For edge detection, a set of 16 fuzzy templates [18] each of size $3 \times 3$, representing the edge profiles of different types, have been used. The choice of templates is crucial which reflects the type and direction of edges It is observed that on increasing the number of templates beyond 16 , there is no remarkable change in the edge results and on decreasing the number of templates, many edges were found missing. The values of $a=0.3$ and $b=0.8$ are chosen by trial and error method. The center of each template is placed at each position $(i, j)$ over a normalized image. The IFD measure at each pixel position ( $i, j)$, in the image, where the template was centered, IFD $(i, j)$, is calculated between the image window and the template using the max-min relationship.

The first algorithm includes the following steps:

1. A set of 16 fuzzy templates with values ' $a$ ' and ' $b$ ' are considered.

2. By placing the center of each template at each point $(i, j)$ over the normalized image, the edge templates are applied over the image.

3. Divergence between each template and image window is calculated.

4. All the sixteen $3 \times 3$ templates are processed and then the maximum of all the 16 minimum intuitionistic fuzzy divergence values is chosen.

5. Position the maximum value at the point where the template was centered over the image.

6. The max-min value is selected and positioned for all the pixel positions.

Finally IFD matrix, the same size as that of image, is formed with values of IFD $(i, j)$ at each point of the matrix. This IFD matrix is thresholded and thinned to get an edge detected image. Then the detected edge points are linked by thresholding the edge image to remove weak edge points. The edge strength associated with compatible edge is increased and the edge strength associated with incompatible edge is decreased.

\subsection{Second Algorithm}

This algorithm relies on the image gradient to locate breaks in uniform regions. An image gradient is a directional change in the intensity or color in an image. Gradient images are obtained from the original image by convolution operation. Each pixel of a gradient image measures the change in intensity of that same point in the original image, in a given direction. To acquire the full range of the direction, gradient images in the $\mathrm{x}$ and $\mathrm{y}$ directions are computed. After the 
gradient images have been computed, the pixels with largest gradient values become edge pixels, and edges may be traced in the direction perpendicular to the gradient direction.

\subsubsection{Membership Functions}

The fuzzy logic approach for edge linking allows to use membership functions to define the degree to which a pixel belongs to an edge or a uniform region. A zero-mean Gaussian membership function is specified for each input of the edge Fuzzy Interference system (FIS). If the gradient value for a pixel is ' 0 ' then it belongs to zero membership function with a degree of 1 . Standard deviations for the zero membership can be changed to adjust the performance of edge detector. Increasing the values makes the algorithm less sensitive to edges in the image and decreases the intensity of detected edges. Triangular membership functions, 'white' and 'black' are specified for the output of edge FIS i.e. $\mathrm{I}_{\text {out }}$. Membership function plots for inputs and outputs of edge FIS are shown in figure 1.
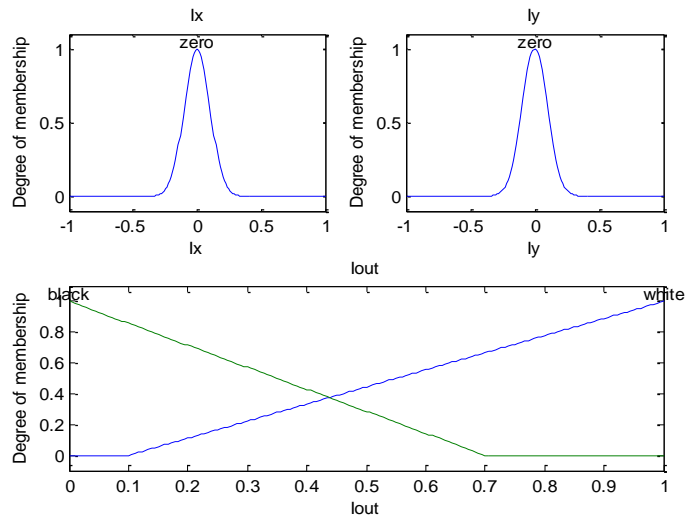

Figure 1: Membership functions for zero,white and black

The second algorithm includes the following steps:

1.Import the filtered RGB image and convert to grayscale image.

2. Calculate the image gradients along the $\mathrm{x}$-axis and $\mathrm{y}$-axis

3. Create a Fuzzy Interference system(FIS) for edge detection.

4. Specify the image gradients, $I_{x}$ and $I_{y}$, as inputs of edge FIS.

5. Specify the intensity of edge-detected image as an output of edge FIS.

6. Specify rules for Fuzzy Interference system (FIS) to make a pixel black or white.

Rule 1: 'If $I_{x}$ is zero and $I_{y}$ is zero, then $I_{\text {out }}$ is white'

Rule 2: 'If $I_{x}$ is not zero or $I_{y}$ is not zero then $I_{\text {out }}$ is black'

7. Evaluate the output of FIS for each row of pixels in the image using corresponding rows of $\mathrm{I}_{\mathrm{x}}$ and $\mathrm{I}_{\mathrm{y}}$ as inputs.

Edges are detected using FIS, by comparing the gradient of every pixel in $\mathrm{x}$ and $\mathrm{y}$ directions. If the gradient for a pixel is not zero then the pixel belongs to an edge (black). For linking the detected edge pixels, hysteresis thresholding is used. This performs a dual thresholding operation to identify strong and weak edge points. This uses a loop to produce a more connect segmentation with fewer isolated pixels. Eight connectivity is used.

\section{SIMULATION RESULTS}

As a measure of comparison between the filtered image and edge linked one, peak signal-to-noise ratio (PSNR) in decibels $(\mathrm{dB})$ is used.

$\operatorname{PSNR}(\mathrm{dB})=10 * \log \left(255^{\wedge} 2 / \mathrm{MSE}\right)$

Where MSE is the Mean Square Error. The PSNR values of the filtered image before and after applying the two fuzzy edge linking algorithms are shown in table 1 for various noise levels. Figure 2 to Figure 10 shows the visual observations for the results tabulated in table- 1 . From the numerical results in table-1, it can be observed that for standard deviation of ' 3 ' the PSNR value increased from $33 \mathrm{~dB}$ to $54 \mathrm{~dB}$ for the first algorithm and to $52 \mathrm{~dB}$ for the second algorithm.

\section{Table-1}

Comparison of PSNR of the filtered image before and after applying the proposed fuzzy edge linking algorithm-1 and algorithm-2 for the $(512 \times 512)$ colored Lena image

\begin{tabular}{|c|c|c|c|}
\hline \multicolumn{4}{|c|}{ PSNR(dB) } \\
\hline $\boldsymbol{\sigma}$ & $\begin{array}{c}\text { Filtered } \\
\text { image } \\
\text { before edge } \\
\text { linking }\end{array}$ & $\begin{array}{c}\text { Output image } \\
\text { after applying } \\
\text { fuzzy edge } \\
\text { linking } \\
\text { algorithm-1 }\end{array}$ & $\begin{array}{c}\text { Output image } \\
\text { after applying } \\
\text { fuzzy edge } \\
\text { linking } \\
\text { algorithm-2 }\end{array}$ \\
\hline 3 & 33.5824 & 54.1962 & 52.4075 \\
\hline 5 & 32.7361 & 54.1601 & 52.3941 \\
\hline 10 & 30.6698 & 53.2548 & 51.5841 \\
\hline 15 & 28.6341 & 52.3609 & 51.0156 \\
\hline
\end{tabular}

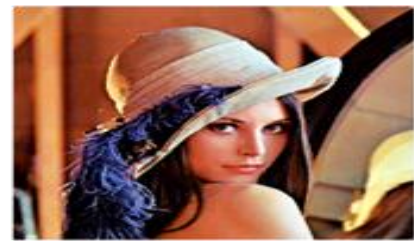

Figure 2: Original Lena image

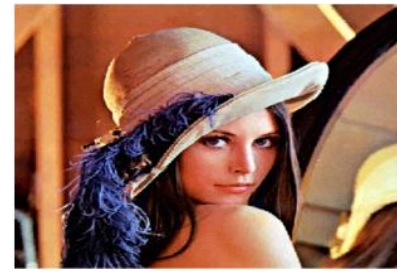

(a) Noisy image

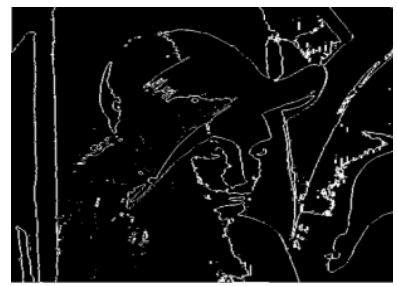

(c) Edge detected image

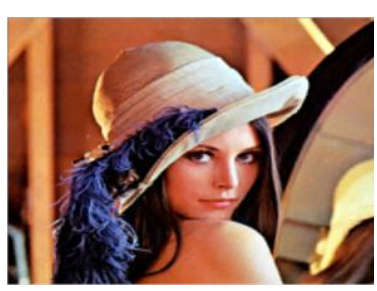

(b) Filtered image

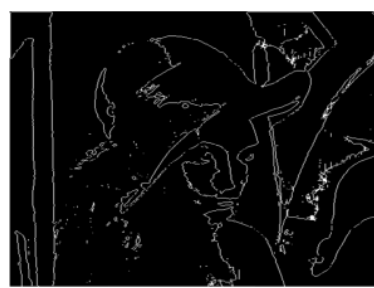

(d) Edge linked image
Figure 3: Illustration of fuzzy edge linking algorithm-1results for Lena image with $\sigma=3$ 


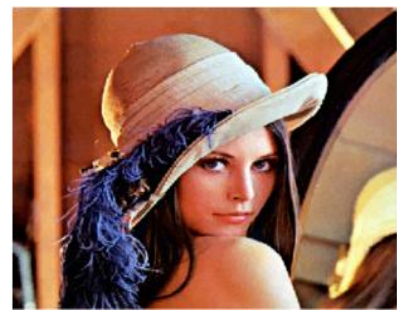

(a) Noisy image

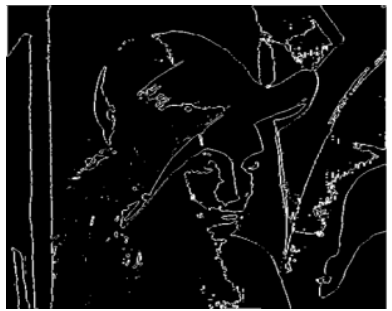

(c) Edge detected image

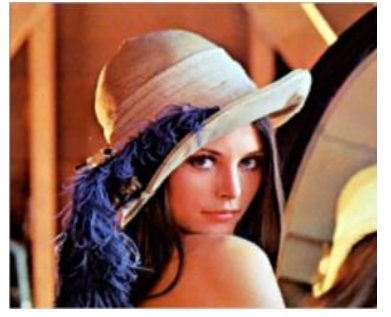

(b) Filtered image

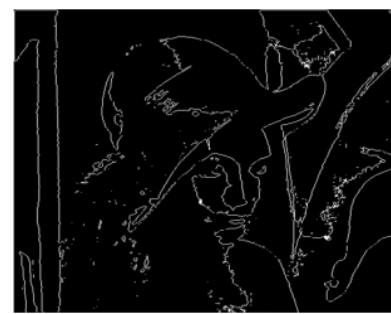

(d) Edge linked image

Figure 4: Illustration of fuzzy edge linking algorithm-1results for Lena image with $\sigma=5$

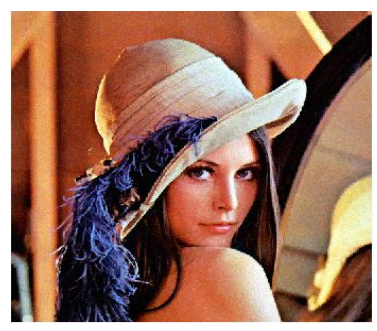

(a) Noisy image

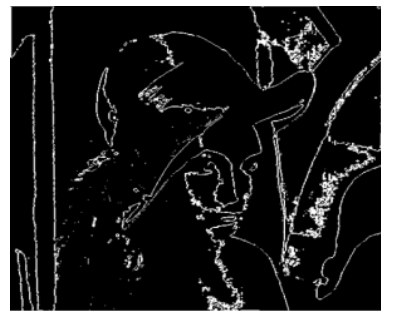

(c) Edge detected image

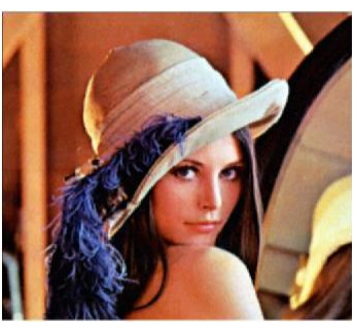

(b) Filtered image

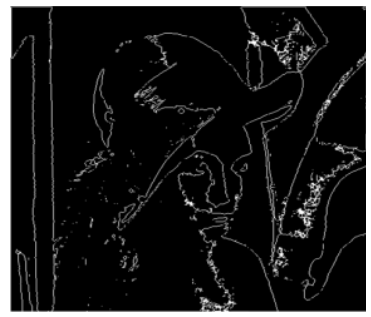

(d) Edge linked image
Figure 5: Illustration of fuzzy edge linking algorithm -1 results for Lena image with $\sigma=10$

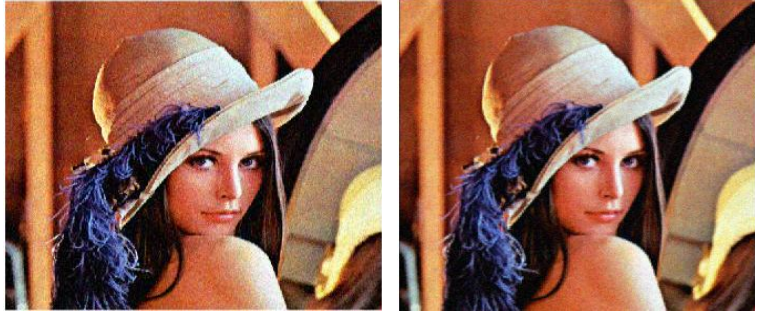

(a) Noisy image

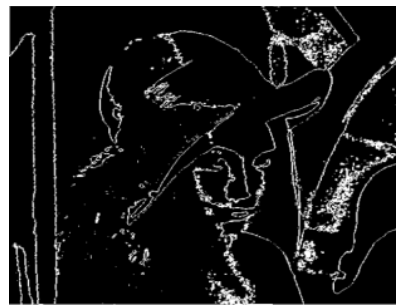

(c) Edge detected image (b) Filtered image

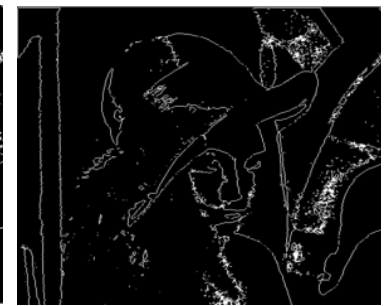

(d) Edge linked image
Figure 6: Illustration of fuzzy edge linking algorithm-1 results for Lena image with $\sigma=15$

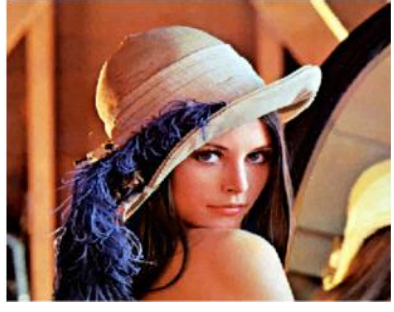

(a) Noisy image

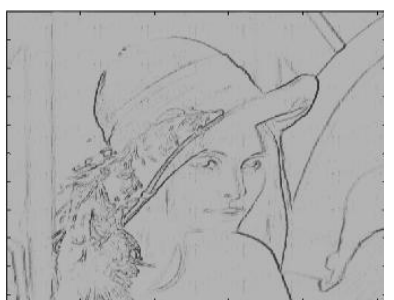

(c) Edge detected image

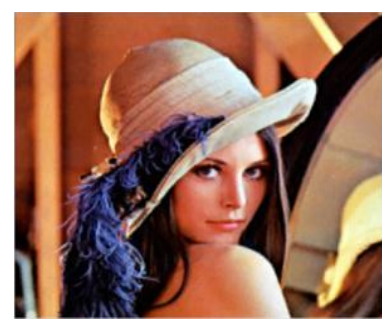

(b) Filtered image

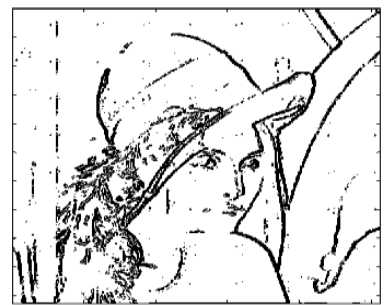

(d) Edge linked image
Figure 7: Illustration of fuzzy edge linking algorithm-2 results for Lena image with $\sigma=3$ 


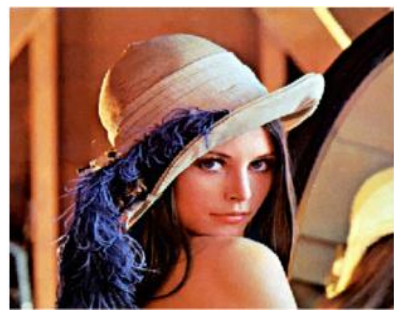

(a) Noisy image

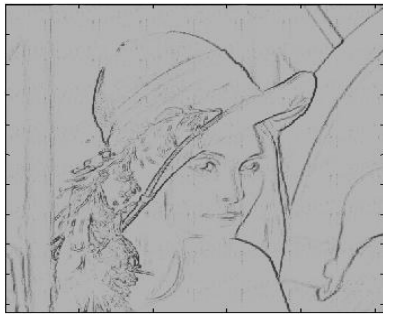

(c) Edge detected image

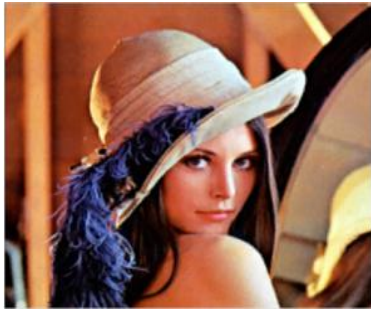

(b) Filtered image

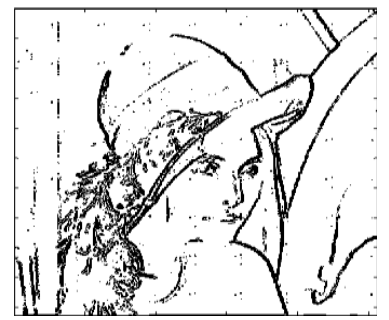

(d) Edge linked image
Figure 8: Illustration of fuzzy edge linking algorithm-2 results for Lena image with $\sigma=5$

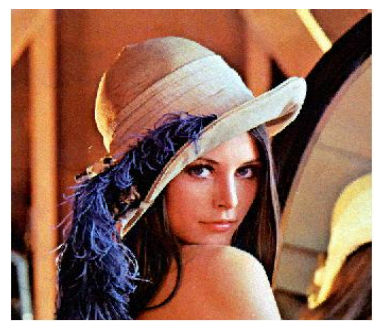

(a) Noisy image

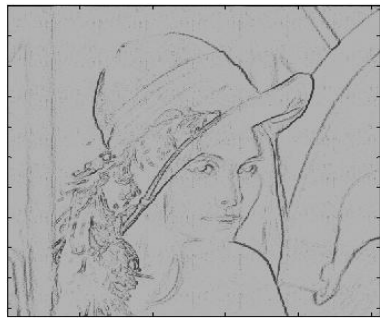

(c) Edge detected image

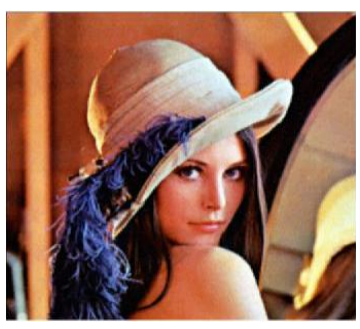

(b) Filtered image

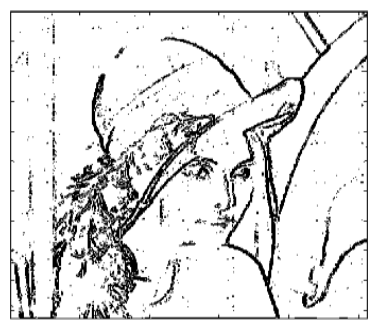

(d) Edge linked image
Figure 9: Illustration of fuzzy edge linking algorithm-2 results for Lena image with $\sigma=10$

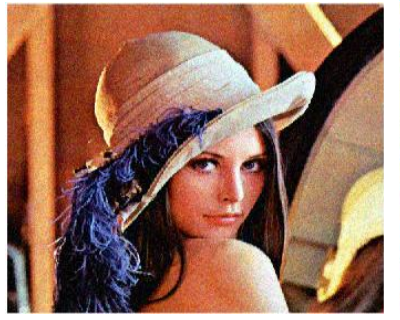

(a) Noisy image

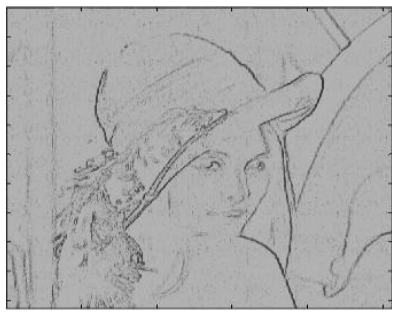

(c) Edge detected image

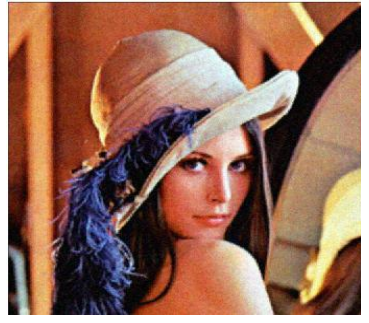

(b) Filtered image

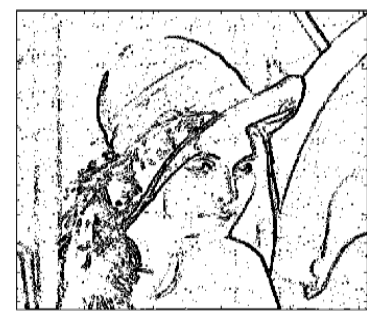

(d) Edge linked image
Figure 10: Illustration of fuzzy edge linking algorithm-2 results for Lena image with $\sigma=15$

\section{CONCLUSION}

This paper proposed two fuzzy edge linking algorithms for fine recovery of edges of the images. These algorithms are applied on the output image of the fuzzy noise filter in a parallel way. Numerical measures and visual observations have shown convincing results. Even at higher levels of noise these two algorithms have shown fine recovery of edges of the images. By observations from table- 1 , it is clear that there is an improvement in the peak signal-to-noise ratio (PSNR) of the filtered image after applying the proposed two fuzzy edge linking algorithms.

\section{REFERENCES}

[1] E. E. Kerre, Fuzzy sets and Approximate Reasoning, Xian, China: Xian Jiaotong Univ.Press, 1998

[2] Farzam Farbiz, Mohammad Bager Menhaz, Seyed A.Motamedi, and Martin T. Hagan "A New Fuzzy Logic Filter for Image Enhancement" IEEE TRANSACTIONS ON SYSTEMS, MAN, AND CYBERNETICS - PART B: CYBERNETICS, VOL. 30, NO. 1, FEBRUARY 2000.

[3] How - Lung Eng, Student Member, IEEE, and Kai-Kuang Ma "Noise Adaptive Soft-Switching Median Filter" IEEE TRANSACTIONS ON IMAGE PROCESSING, VOL. 10, NO. 2, FEBRUARY 2001.

[4] Dimitri Van De Ville, Mike Nachtegael, Dietrich Van der Weken, Etienne E. Kerre, Wilfried Philipsand Ignace Lemahieu "Noise Reduction by Fuzzy Image Filtering" IEEE TRANSACTIONS ON FUZZY SYSTEMS, VOL. 11, NO. 4, AUGUST 2003.

[5] Stefan Schulte, Valerie De Witte, Mike Nachtegael, Dietrich Van der Weken, and Etienne E.Kerre "Fuzzy Two-Step Filter for Impulse Noise Reduction From Color Images" IEEE TRANSACTIONS ON IMAGE PROCESSING, VOL. 15, NO. 11, NOVEMBER 2006. 
[6] Stefan Schulte, Mike Nachtegael, Valerie De Witte, Dietrich Van der Weken, and Etienne E. Kerre "A Fuzzy Impulse Noise Detection and Reduction Method" IEEE TRANSACTIONS ON IMAGE PROCESSING, VOL.15, NO. 5, MAY 2006.

[7] Stefan Schulte "Fuzzy and Nonlinear Restoration and Analysis Techniques for Digital Images".

[8] Tom Melange, Mike Nachtegael, and Etienne E. Kerre "Fuzzy Random Impulse Noise Removal from Color Image Sequences" IEEE TRANSACTIONS ON IMAGE PROCESSING, VOL. 20, NO. 4, APRIL 2011.

[9] G. Sudhavani and K. Satya Prasad "Segmentation of Lip Images by Modified Fuzzy C- means Clustering Algorithm" International Journal of Computer Science \& Network Security, Vol. 9 No. 4, April 2009.

[10] "FUZZY MODELS AND ALGORITHMS FOR PATTERN RECOGNITION AND IMAGE PROCESSING" by James C. Bezdek, James Keller, Raghu Krisnapuram, Nikhil R. Pal.

[11] T. Chaira, Image Segmentation and Color Retrieval-A Fuzzy and Intuitionistic Fuzzy Set Theoretic Approach, $\mathrm{PhD}$ Thesis, Indian Institute of Technology, Kharagpur.India, 2004.
[12] T. Chaira, A.K. Ray, Segmentation using fuzzy divergence, Pattern Recog. Lett. 24 (12) (2003) 1837 1844.

[13] C.Vertan and V. Buzuloiu, "Fuzzy nonlinear filtering of color images," in Fuzzy Techniques in Image Processing, E. E. Kerre and M. Nachtegael, Eds., 1st ed. Heidelverg, Germany: Physica Verlag, 2000, vol. 52, pp. 248-264

[14] S. M. Guo, C. S. Lee, and C. Y. Hsu, "An intelligent image agent based on soft-computing techniques for color image processing," Expert Sist Appl., vol. 28, pp.483-494, Apr. 2005.

[15] T. Takagi and M. Suguno, "Fuzzy identification of systems and its applications to modeling and control," IEEE Trans. Syst., Man, Cybern., vol. SMC-15, no. 1, pp. 116-132, Jan. 1985.

[16] G. Sudhavani, G. Madhuri, P. Venkateswa Rao \& K. Satya Prasad "Removing Gaussian Noise from Color Images by Varying the Size of Fuzzy Filters" International Journal of Computer Applications (0975 8887), Vol. 72, No. 17, June 2013.

[17] K.T. Atanassov, Intuitionistic fuzzy set. Fuzzy Sets Syst. (1986) 87-97.

[18] "Computational Intelligence in Medical Informatics" by Arpad Kelemen, Ajith Abraham .Yulan Liang (Eds.) 